\title{
Comportamiento del consumidor en el uso de la biblioteca virtual de acceso libre. Un caso de los estudiantes de la Universidad Nacional del Altiplano, Perú
}

\author{
Emilio Flores ${ }^{1}$, Wilber C. Calsina ${ }^{2}$ y Benjamin Velazco ${ }^{3}$ \\ (1) Facultad de Ciencias Sociales, Dpto. Ciencias de la Comunicación Social, Universidad Nacional del Altiplano de Puno, \\ Av. Floral S/N, Puno-Perú. (correo-e: emilioflores@unap.edu.pe) \\ (2) Facultad de Ciencias Sociales, Dpto. de Arte, Universidad Nacional del Altiplano de Puno, Av. Floral S/N Puno-Perú \\ (correo-e: wcalsina@unap.edu.pe) \\ (3) Facultad de Ciencias Sociales, Dpto. de Arte, Universidad Nacional del Altiplano de Puno, Av. Floral S/N, Puno-Perú \\ (correo-e: bvelazco@unap.edu.pe)
}

Recibido Oct. 21, 2020; Aceptado Dic. 18, 2020; Versión final Ene. 12, 2021, Publicado Jun. 2021

\begin{abstract}
Resumen
El objetivo del presente estudio es analizar el comportamiento del consumidor respecto a la influencia de facilidad y rapidez en la obtención de información virtual para la elaboración de trabajos académicos en la Universidad Nacional del Altiplano (Perú). El enfoque es cuantitativo y el tipo de investigación es descriptiva, explicativa y de diseño no experimental. La muestra de estudio se obtuvo proporcionalmente de cada escuela profesional. Se utiliza la técnica de encuesta escala de Likert en 5 categorías: muy deficiente, deficiente, regular, bueno y excelente. Los resultados muestran que solo en $17 \%$ influyen los criterios de selección de información virtual, análisis crítico y reflexión de los temas en la elaboración de los trabajos académicos. Se concluye que los estudiantes adquirieron los conocimientos de buena conducta en investigación formativa, por lo que en sus trabajos académicos muestran un uso correcto de las fuentes utilizadas.
\end{abstract}

Palabras clave: usuario de biblioteca; información virtual; trabajo académico; disponibilidad de información

\section{User behavior in a free access virtual library. A case study of students at the National University of Altiplano, Peru}

\begin{abstract}
The main objective of the present study is to assess user behavior and the influence of ease and speed when users obtain information virtually for academic assignments at the National University of the Altiplano (Peru). The research is quantitative, descriptive, explanatory, and non-experimental in design. The Likert scale is used for data collection and is arranged in five different categories: very deficient, deficient, regular, good, and excellent. The results show that in only $17 \%$ of the time, virtual information selection criteria and critical analysis and reflection of a given topic influence the preparation of academic assignments. It is concluded that students have acquired proper research practices, which explains why their academic assignments show correct utilization of the resources used.
\end{abstract}




\section{INTRODUCCION}

Nuevas directrices para el acceso a la información son utilizadas en el modelo de biblioteca digital, debido al desarrollo de la Web 2.0 para producir, consumir y compartir información a través de las redes que se constituyen relevantes en el sistemas de información digital abierta y permite lograr la convergencia cultural en el contexto (Rodríguez-García, 2016). Es complejo, pero, se entiende al consumidor como individuos o grupos que seleccionan, compran, usan o disponen de productos, servicios, ideas o experiencias para satisfacer las necesidades y deseos (Silva et al., 2010). Sin embargo, el comportamiento de los usuarios de las bibliotecas universitarias con la presencia de la tecnología de las comunicaciones ha cambiado vertiginosamente en su forma de usarla para trabajos académicos, sobresaliendo la preferencia de las fuentes no necesariamente las más confiables, sino por la facilidad y rapidez de encontrarlo.

El desarrollo vertiginoso de la tecnología ha permitido la virtualización de las bibliotecas de consulta a través de la red o el internet sin considerar las fronteras físicas, constituyéndose para los estudiantes en un medio fácil y rápido para proveerse de información. Bashorun et al. (2016) la aparición de la World Wide Web (www) ha cambiado el sistema de comunicaciones académicas consistente en la publicación de acceso abierto que ofrece un nuevo modelo de comunicación para académicos, investigadores, estudiantes y otras personas interesadas. En dicho contexto, la web tiene una gran importancia, ya que a través de la misma se le ofrece al usuario acceso directo o diferido a todos los recursos y servicios (Camacho, 2004).

Las webs de bibliotecas escolares de Andalucía y Extremadura de España que permite acceder directamente a las fuentes (Jiménez, 2013). Se ha constatado mayor incidencia de herramientas y tecnologías online 2.0 en las bibliotecas universitarias en los servicios de referencia y atención al usuario y, las herramientas más utilizadas fueron redes sociales, blogs y chats (Xavier y Godoy, 2010). Por lo tanto, la biblioteca virtual es una estrategia que promueve el acceso abierto, arbitrado, equitativo y de calidad a la información (Niño, 2016). Además, ofrece a los jóvenes independencia e intervención en la búsqueda y uso de la información (Kasman, 2006).

Antes, los libros de las bibliotecas tradicionales universitarias mostraban signos de uso frecuente por parte de los estudiantes, quienes eran obligados a leer y entender para sus trabajos académicos, por eso los ambientes de las bibliotecas físicas permanecían repletos durante todo el día, donde se desarrollaba la capacidad de análisis crítico y comprensión de la obra y que finalmente le permitía abstraer las conclusiones. Yucra (2011) los estudiantes dedicaban tiempo extra para sus estudios y cumplir con sus deberes académicos como trabajos encargados, presentación de exposiciones y otros. No obstante, la presencia de internet fue fundamental para facilitar información al estudiante en sus trabajos, se constituyó en una herramienta de enorme utilidad para la obtención e intercambio de información (Sanz-Valero et al., 2008).

Sin embargo, las bibliotecas virtuales o bases de datos de acceso libre son benevolentes con los usuarios por la facilidad y rapidez que brindan información como recurso para realizar sus trabajos académicos, en vista que ofrece diversidad de información digitalizada. Aguilar y Moctezuma (2020) el proceso de búsqueda virtual se convierte en esencial en un entorno donde la información se multiplica y se diversifica en diferentes formatos. Estas informaciones no suelen ser tratadas y procesadas para abstraer información sustancial en la asimilación del contenido, sino, los hábitos de buen estudio son remplazados por actitudes facilistas de obtener información en tiempo récord a través de la red, sin considerar el protocolo del uso de las fuentes, utilizando tal cual como se ofrece en la red, de esa forma algunos usuarios violentan los derechos del autor. Así como reveló el estudio donde más del $80 \%$ de los estudiantes universitarios que reconocen haber cometido actos de este tipo a lo largo de su carrera (Marsden, Carroll y Neill, 2005).

El derecho de autor es considerado una parte moral intransferible y una parte patrimonial, donde el autor tiene el derecho de percibir parte de los ingresos económicos derivados del uso público de la creación literaria o artística (Romero, 2007). Pero, la forma más correcta de valorar y reconocer a los autores es citándolos cuando se hace uso de sus ideas, frases o texto en la reconstrucción de los trabajos académicos universitarios. Caso contrario, se daría la deshonestidad académica, expresada como plagio, el cual es definido como la presentación intencional del trabajo o partes del trabajo de otra persona, como producción propia, sin el reconocimiento apropiado de la fuente (Moeck, 2002). El objetivo del artículo es analizar el comportamiento del consumidor respecto a la influencia de la facilidad y rapidez de obtención de la información virtual en la elaboración de trabajos académicos de los estudiantes de la Universidad Nacional del Altiplano - Perú en el año 2019.

\section{METODOLOGIA}

La investigación se desarrolló en el enfoque cuantitativo, diseño no experimental y transeccional, tipo de investigación descriptivo y explicativo. La técnica de recolección de datos fue la encuesta, elaborada considerando los objetivos del estudio, siendo el instrumento la escala de Likert para recoger información a 
partir de las dimensiones e indicadores. Se elaboraron 2 preguntas generales y 35 afirmaciones específicas sobre indicadores del estudio con escalas de: nunca $=1$, casi nunca $=2$, a veces $=3$, casi siempre $=4$ y siempre $=5$. Para cada objetivo especifico de la investigación se elaboró un conjunto de afirmaciones.

La población de estudio fueron los estudiantes de pre grado que suman más de 18 mil y la muestra representativa ascendió a 384 estudiantes entre varones y mujeres de todos los semestres académicos, la cual fue hallada a través del muestreo estratificado. La unidad de análisis es el comportamiento de los estudiantes respecto al uso de la biblioteca virtual. La recolección de datos se obtuvo según la cantidad de facultades y escuelas profesionales con que cuenta la universidad. Considerando la proporcionalidad que corresponde a cada escuela profesional y al azar, los mismos que no fueron del mismo semestre académico. Además, el horario de recolección fue en el turno de la mañana y en la tarde, a fin de garantizar una información objetiva.

Asimismo, la encuesta previamente fue sometida a la prueba piloto a través del alfa de Crombah al $10 \%$ que corresponde a 38 estudiantes, para garantizar la confiabilidad del estudio, cuyo resultado fue 0.908 que nos permitió continuar. Por otro lado, la encuesta fue validada por dos expertos. Se tabularon y se analizaron los datos recogidos en el software IBM SPSS Statistics 26.0, a fin de explicar por objetivos mediante la estadística descriptiva e inferencial para determinar el patrón de comportamiento del usuario estudiantil en el uso de la biblioteca virtual de acceso libre y la respectiva influencia de la variable independiente.

Para analizar los datos obtenidos se transformó y se calculó en variables agrupados tales como: razones de uso de la biblioteca virtual, internet como ayuda, valoración de autores y trabajos académicos y luego se hizo la valoración en categorías de muy deficiente, deficiente, regular, bueno y excelente para interpretar y llegar a las conclusiones. Los resultados obtenidos fueron discutidos con autores consultados como antecedentes y otros a fin de encontrar las diferencias y similitudes existentes con el estudio, así como, se resaltó los limitantes que no permitieron la objetividad completa del estudio.

\section{RESULTADOS Y DISCUSIÓN}

En la tabla 1 se muestra que el 5,5\% de estudiantes hace uso de la biblioteca virtual todos los días de la semana; mientras que $21,6 \%$, una vez en el semestre, siendo otros intermedios que lo caracterizan el uso de materiales bibliográficos virtuales en sus trabajos académicos. La biblioteca en términos tradicionales es un lugar de convergencia de diferentes tipos de estudiantes que hacen uso de la bibliografía y es un lugar donde se construye la amistad. Meneses (2013) resalta como una institución social donde no se puede ignorar la relación biblioteca y sociedad en general y biblioteca pública y sociedad. Entonces la biblioteca también es un lugar de socialización entre los estudiantes y otros usuarios.

Tabla 1: Frecuencia de uso de la biblioteca virtual

\begin{tabular}{|l|l|c|c|c|c|}
\hline \multicolumn{2}{|l|}{} & Frecuencia & Porcentaje & $\begin{array}{c}\text { Porcentaje } \\
\text { válido }\end{array}$ & $\begin{array}{c}\text { Porcentaje } \\
\text { acumulado }\end{array}$ \\
\hline Válido & Una vez en el semestre & 83 & 21,6 & 21,6 & 21,6 \\
\hline & Una vez al mes & 68 & 17,7 & 17,7 & 39,3 \\
\hline & Dos veces al mes & 82 & 21,4 & 21,4 & 60,7 \\
\hline & Una vez a la semana & 130 & 33,9 & 33,9 & 94,5 \\
\hline & Todos los días de la semana & 21 & 5,5 & 5,5 & 100,0 \\
\hline
\end{tabular}

Hernández, Palomo y Cantú (2013) encontraron el 40,3\% de estudiantes que hace uso de la biblioteca digital una vez al semestre y el $10,3 \%$ todos los días. Este último dato duplica a los datos obtenidos en el estudio. Pero, en sentido comparativo de ambos estudios se deduce que aún son todavía pocos los que tienen el hábito de hacer uso diariamente de la biblioteca virtual, la que podría atribuirse al escaso recurso que cuentan la mayoría de los estudiantes de universidades públicas. El otro gran segmento apenas hace uso de la biblioteca virtual una vez en el semestre; esto significa que ellos frecuentan la visita a la biblioteca tradicional donde utilizan las técnicas tradicionales de comprensión y abstracción, evitando de esa forma los problemas de facilismo en la obtención de la información requerida.

La Tabla 2, muestra que el 60,9\% manifiesta que hace uso ambas fuentes, virtual y física; en tanto que el $16,4 \%$ solamente hacen uso de la biblioteca virtual y 21,9 usan puro libros físicos. Estos datos permiten conocer sobre el comportamiento de los estudiantes respecto a la preferencia en el uso de la bibliografía física o virtual. Donde se impone el uso habitual de ambas fuentes. 
Tabla 2: Tipo de biblioteca más usado para hacer su trabajo académico

\begin{tabular}{|l|l|c|c|c|c|}
\hline \multicolumn{2}{|c|}{} & Frecuencia & Porcentaje & $\begin{array}{c}\text { Porcentaje } \\
\text { válido }\end{array}$ & $\begin{array}{c}\text { Porcentaje } \\
\text { acumulado }\end{array}$ \\
\hline \multirow{5}{*}{ Válido } & Biblioteca física (solamente libros). & 84 & 21,9 & 21,9 & 21,9 \\
\cline { 2 - 6 } & $\begin{array}{l}\text { Biblioteca física (libros) y virtual (internet páginas } \\
\text { web, buscadores). }\end{array}$ & 234 & 60,9 & 60,9 & 82,8 \\
\cline { 2 - 6 } & Solo biblioteca virtual (página web, buscadores) & 63 & 16,4 & 16,4 & 99,2 \\
\hline & 4 & 2 &, 5 &, 5 & 99,7 \\
\hline & 5 & 1 &, 3 &, 3 & 100,0 \\
\cline { 2 - 6 } & Total & 384 & 100,0 & 100,0 & \\
\hline
\end{tabular}

Una forma de sacar ventaja en el uso de las fuentes físicas y virtuales que es el porcentaje más alto, debe partir desde las políticas institucionales universitarias para el uso responsable de la bibliografía. Debe ser políticas públicas educativas de buen uso del material en el proceso de enseñanza - aprendizaje desde el más alto nivel hasta el salón de clases (Cárdenas, 2012; Carter, Hussey y Forehand, 2019). El comportamiento y actitudes de los estudiantes de post grado de Latino América y Estados Unidos, muestran comodidad y confianza al encontrar información, tanto en forma impresa como electrónica, pero, los segundos prefieren la electrónica por la facilidad (Mazurkiewicz y Sturm, 2014). En ese panorama es importante formar estudiantes con ética y valores en la elaboración de sus trabajos académicos, respetando los derechos del autor y garantizar un producto original que tenga su inicio desde la lectura, análisis crítico y reflexión sobre el tema de estudio. Se debe privilegiar la honestidad en la formación ético profesional, la responsabilidad, el respeto y el compromiso social (Osuna y Luna, 2011).

Cuando se hace referencia al 16,4\% que solo hacen uso de la biblioteca virtual se puede dar crédito a (Xavier y Godoy, 2010) quienes dicen que existe mayor incidencia de herramientas y tecnologías online 2.0 en las bibliotecas universitarias, las herramientas más utilizadas son redes sociales, Blogs y Chats que son empleadas para comunicarse, excepto los blog que contienen material bibliográfico de consulta. Asimismo, corrobora el estudio donde revela que el 92,4\% de los encuestados había utilizado información electrónica, de los cuales el 51,8\% fueron usuarios frecuentes; los principales recursos electrónicos utilizados figuran motores de búsqueda de Internet, notas de clase y libros electrónicos empleados para completar las tareas de la clase, obtener materiales relacionados con el curso y para mantenerse al tanto de los últimos avances en su campo (Adeniran, 2013). Pero, los estudiantes no necesariamente pueden utilizar las bases de datos de accedo libre con validez científica, porque algunas universidades no tienen políticas de acceso abierto para motivar la adopción y el uso de académicos tal como revelaron (Bashorun et al., 2016). Por lo tanto, se debe sensibilizar a los estudiantes a proveerse del material bibliográfico virtual de fuentes con validez científica, como son las bases de datos en donde se encuentran revistas indizadas y arbitrada por pares.

Respecto a las razones del uso de la biblioteca virtual, la tabla 3, muestra que el 50,3 \% manifiesta que sus razones son buenos y $32,3 \%$ usa razones de forma excelente para realizar sus actividades cotidianas utilizando información virtual. Siendo un acumulado de 82,6 \% que utilizan justificativamente la biblioteca virtual para realizar sus trabajos académicos.

Tabla 3: Razones de uso de la biblioteca virtual (Agrupada)

\begin{tabular}{|l|l|c|c|c|c|}
\hline \multicolumn{2}{|c|}{} & Frecuencia & Porcentaje & Porcentaje válido & $\begin{array}{c}\text { Porcentaje } \\
\text { acumulado }\end{array}$ \\
\hline \multirow{4}{*}{ Válido } & Muy deficiente & 3 &, 8 &, 8 &, 8 \\
\cline { 2 - 6 } & Deficiente & 6 & 1,6 & 1,6 & 2,3 \\
\cline { 2 - 6 } & Regular & 58 & 15,1 & 15,1 & 17,4 \\
\cline { 2 - 6 } & Bueno & 193 & 50,3 & 50,3 & 67,7 \\
\cline { 2 - 6 } & Excelente & 124 & 32,3 & 32,3 & 100,0 \\
\cline { 2 - 6 } & Total & 384 & 100,0 & 100,0 & \\
\hline
\end{tabular}

La formación universitaria requiere de material bibliográfico con validez científica para realizar los trabajos académicos. (Golitsyna, 2017) la web se convierte en una herramienta importante de provisión de material bibliográfico confiable para las actividades de aprendizaje de los estudiantes, su uso contribuye al desarrollo de competencias clave a nivel profesional de acuerdo con los estándares educativos de la nueva generación. 
Puede ser un medio extraordinario para la participación de los miembros de la comunidad educativa en el uso de los recursos informativos y de comunicación (Camacho, 2004). Por tanto, las razones de uso de las fuentes virtuales por parte de los estudiantes se justifican debido a la confiabilidad y conocimiento con que pueden proveerse para realización de sus trabajos académicos y para su mejor formación profesional. Este tipo de biblioteca es formada como una red de colaboración entre productores, intermediarios y usuarios (Niño, 2016). La biblioteca debe hacer que la lectura se despliegue en una diversidad de posibilidades, así los sujetos se formarían en la medida en que potencian sus capacidades y se hacen responsables de su desarrollo y con ello, de su destino (Ramírez-Leyva, 2016). Como resaltan los autores, las bibliotecas y bibliografías permiten la interrelación entre los sujetos de la cadena bibliográfica y también potenciar las capacidades de raciocinio y creatividad en su formación profesional de los estudiantes universitarios.

La tabla 4 muestra que el internet es una gran ayuda para la elaboración de trabajos académicos. El $67 \%$ manifiesta como buena ayuda para realizar sus trabajos, mientras el $24 \%$ califica excelente, en vista de proveerse con facilidad y en un tiempo récord el material requerido y el 0,3\% deficiente. Camacho (2004) confirma que, en la biblioteca, la web tiene una gran importancia, puesto que se le ofrece al usuario acceso directo o diferido a todos los recursos y servicios. Carera, Delgado y contreras (2017) encuentran la eficiencia en la búsqueda de información en un $37,23 \%$ de usuarios. La información virtual es efectivamente una gran ayuda para proveerse de material y realizar trabajos académicos en un tiempo récord. Pero, cuando se hace reflexión y análisis respectivo sobre el contenido de las fuentes, obviamente demanda más tiempo en la reconstrucción de dicho conocimiento, es el 0,3\% que practica esta forma de estudio.

Tabla 4: Internet como ayuda (Agrupada)

\begin{tabular}{|l|l|c|c|c|c|}
\hline \multicolumn{2}{|c|}{} & Frecuencia & Porcentaje & Porcentaje válido & $\begin{array}{c}\text { Porcentaje } \\
\text { acumulado }\end{array}$ \\
\hline \multirow{4}{*}{ Válido } & Deficiente & 1 &, 3 &, 3 &, 3 \\
\cline { 2 - 6 } & Regular & 32 & 8,3 & 8,3 & 8,6 \\
\cline { 2 - 6 } & Bueno & 259 & 67,4 & 67,4 & 76,0 \\
\cline { 2 - 6 } & Excelente & 92 & 24,0 & 24,0 & 100,0 \\
\cline { 2 - 6 } & Total & 384 & 100,0 & 100,0 & \\
\hline
\end{tabular}

Los hábitos de lectura para realizar los trabajos académicos muchas veces son sustituidos por transcripciones literales de ideas o texto de autores, el plagio, en vista que es más fácil y rápido copiar y pegar que leer el texto completo, analizar sobre lo que piensan los autores y finalmente hacer la abstracción como conclusiones. Este tipo de hábitos perjudican a los estudiantes en su capacidad de raciocinio, análisis y criticidad que deben tener como futuros profesionales. En consecuencia, la biblioteca virtual tiene la ventaja de provisión rápida de vasto material bibliográfico y desventajas de indiferencia en el análisis y reflexión de los temas por parte de los estudiantes.

En la tabla 5, se puede apreciar que el $62,5 \%$ de estudiantes se ubican dentro de la categoría bueno en el reconocimiento o valoración a autores, mientras que el 13,5\% reconocen de manera excelente a los autores haciendo citas en sus trabajos académicos. Un trabajo original parte desde el uso responsable de las fuentes de consulta, lo cual significa una estrategia de provisión de material virtual a través de revistas arbitradas y con calidad de información (Niño, 2016). Hacer uso de esas fuentes es de restricto respeto y consideración a Ios autores; en los resultados obtenidos un $76 \%$ acumulado reconocen y valoran a los autores.

Tabla 5: Valoración a los autores (Agrupada)

\begin{tabular}{|l|l|c|c|c|c|}
\hline \multicolumn{2}{|l|}{} & Frecuencia & Porcentaje & Porcentaje válido & $\begin{array}{c}\text { Porcentaje } \\
\text { acumulado }\end{array}$ \\
\hline Válido & Deficiente & 3 &, 8 &, 8 &, 8 \\
\hline & Regular & 89 & 23,2 & 23,2 & 24,0 \\
\hline & Bueno & 240 & 62,5 & 62,5 & 86,5 \\
\hline & Excelente & 52 & 13,5 & 13,5 & 100,0 \\
\hline
\end{tabular}

De los resultados se desprende, que los trabajos académicos de los estudiantes de la Universidad Nacional del Altiplano se encuentran dentro de la práctica de los valores de conducta responsable en investigación al elaborar sus trabajos. Excepto el 0,8\% que comete la deshonestidad de plagiar los textos. De esta forma el 
consumo de recursos protegidos por la ley como creación intelectual ligados a la propiedad intelectual requieren respeto a los autores (Romero, 2007). Además, los trabajos académicos pueden tener una connotación de originalidad producto de una reflexión y criticismo que lo realizarían los estudiantes al hacer sus trabajos, lo cual inspiraría confianza en los lectores y puede ser motivo de citaciones en otros trabajos.

En la tabla 6, se observa que de los 383 estudiados el $13 \%$ elabora sus trabajos académicos de manera excelente con el uso de internet y citando los autores; mientras que $64,6 \%$ se ubican dentro del buen uso en la elaboración de sus trabajos. Considerando los porcentajes referidos se puede decir que los estudiantes de la Universidad Nacional del Altiplano que al elaborar sus trabajos académicos reconocen y dan crédito a los autores consultados. De esta forma se tiene presente el derecho de autor, donde el original es considerado inalienable, perpetuo e intransferible (Romero, 2017). Por otro lado, la tecnología moderna: los teléfonos celulares, computadoras e Internet son importantes herramientas de comunicación; pero, en algunos casos han facilitado los actos académicos deshonestos, principalmente el plagio, motivado por la amplia disponibilidad de información en Internet y por las conocidas funciones de "copiar" y "pegar" (Abreu, Domínguez y Fernández, 2011).

Tabla 6: Reconstrucción de trabajos académicos (Agrupada)

\begin{tabular}{|l|l|c|c|c|c|}
\hline \multicolumn{2}{|l|}{} & Frecuencia & Porcentaje & Porcentaje válido & Porcentaje acumulado \\
\hline \multirow{4}{*}{ Válido } & Deficiente & 1 &, 3 &, 3 &, 3 \\
\cline { 2 - 6 } & Regular & 84 & 21,9 & 21,9 & 22,2 \\
\cline { 2 - 6 } & Bueno & 248 & 64,6 & 64,8 & 86,9 \\
\cline { 2 - 6 } & Excelente & 50 & 13,0 & 13,1 & 100,0 \\
\cline { 2 - 6 } & Total & 383 & 99,7 & 100,0 & \\
\hline Perdidos & Sistema & 1 &, 3 & & \\
\hline \multicolumn{1}{|l|}{ Total } & 384 & 100,0 & & \\
\hline
\end{tabular}

A este resultado se atribuye que en el plan de estudios de pregrado de las escuelas profesionales de la Universidad Nacional del Altiplano se contempla los cursos de investigación científica, donde los docentes que administran dichos cursos imparten conocimientos y la práctica de la conducta responsable en investigación. De tal madera que los estudiantes asimilan la forma correcta de redactar los documentos académicos citando las fuentes utilizadas a fin de evitar las falsaciones o plagios.

\section{Influencia de la información virtual en la elaboración de trabajos}

En la tabla 7 y 8 se puede ver que las variables: razones del uso de la biblioteca o el criterio de selección, internet como ayuda para realizar el trabajo académico mediante la crítica, reflexión y el reconocimiento a autores, han influido en la variable elaboración de los trabajos académicos de los estudiantes en $17 \%$. Estos resultados son corroborados con el estudio del uso de la biblioteca virtual en la Universidad de Fountain donde ha mejorado en gran medida la calidad de la enseñanza y la investigación de docentes y estudiantes, así como en sus trabajos académicos (Alijani y Khasseh, 2015). Pero, las principales barreras que pueden obstaculizar el uso eficaz de los recursos electrónicos pueden ser habilidades insuficientes, dificultad para encontrar información relevante y frecuentes cortes de energía (Adeniran, 2013). No solo las dificultades de utilización de los recursos electrónicos pueden atribuirse únicamente al desconocimiento por parte de los estudiantes, a quienes se considera como nativos digitales, sino a factores externos (Mawere y Sai, 2018; Eret y Gokmenoglu, 2010). Esto significa que los usuarios de las bibliotecas virtuales deben tener dominio en el uso de los buscadores para proveerse de materiales bibliográficos para elaborar sus trabajos académicos con fuentes de calidad.

Tabla 7: Resumen del modelo

\begin{tabular}{|c|c|c|c|c|c|c|c|c|c|}
\hline & & & & \multirow{4}{*}{ Modelo } & \multicolumn{4}{|c|}{ Estadísticos de cambio } \\
\cline { 6 - 10 } & $R$ & $R^{2}$ & $R^{2}$ ajustado & Error estándar & Cambio en $R^{2}$ & Cambio en $F$ & gl1 & gl2 & Sig. Cambio en $F$ \\
\hline 1 &, $413^{\mathrm{a}}$ &, 171 &, 164 &, 543 &, 171 & 25,974 & 3 & 379 &, 000 \\
\hline
\end{tabular}


Tabla 8: Resultados Anova

\begin{tabular}{|c|l|c|c|c|c|c|}
\hline \multicolumn{2}{|c|}{ Modelo } & Suma de cuadrados & Gl & Media cuadrática & $F$ & Sig. \\
\hline \multirow{2}{*}{1} & Regresión & 22,957 & 3 & 7,652 & 25,974 &, $000^{\mathrm{b}}$ \\
\cline { 2 - 7 } & Residuo & 111,659 & 379 &, 295 & & \\
\cline { 2 - 7 } & Total & 134,616 & 382 & & & \\
\hline
\end{tabular}

Asimismo, el resultado muestra que las variables mencionadas influyen parcialmente en la elaboración de sus trabajos académicos de los estudiantes de la Universidad Nacional del Altiplano. Comparado con el estudio sobre las influencias de los factores digitales como redes sociales en el rendimiento académico de los estudiantes universitarios y graduados de la ciudad Dhahran, donde la influencia es positiva que alcanza el $61 \%$ (Alasani, Alandejani, 2000). Con dicho estudio existe una diferencia marcada respecto al porcentaje, pero, corrobora a nuestro estudio en el sentido que el uso de la biblioteca virtual sí influye en la realización de actividades académicas de los estudiantes. Sin embargo, es atribuida una correlación con el impulso docente en un $36,51 \%$ y la eficiencia en la búsqueda de información en un 37,23\% (Carera, Delgado y Contreras, 2017). Por lo tanto, existe una aproximación con estos estudios realizados donde se confirman que el uso de la información virtual y su eficiencia de búsqueda, más la participación del docente inciden en la mejor elaboración de los trabajos académicos de los estudiantes, debido al aporte importante de literatura virtual respecto a sus temas de trabajo y que permite complementar su formación profesional.

Finn y Frone (2004) refieren al rendimiento académico de estudiantes secundarios y universitarios y encontraron que los actos de deshonestidad académica son más frecuentes entre los estudiantes con rendimientos bajos y escasa identificación con su institución, así como entre los alumnos con buenos rendimientos, pero reducida autoconfianza académica. Esto quiere decir que los factores como bajo rendimiento, relativa identidad y autoconfianza influyen en la práctica de los plagios o la deshonestidad académica de los estudiantes. No obstante, los hallazgos de la correlación del estudio es que la mayoría de los estudiantes tienen el hábito de hacer sus trabajos académicos según las normas establecidas en producción científica, desde las clases de los cursos de investigación formativa que permite evitar la deshonestidad científica en la elaboración de sus trabajos, excepto el porcentaje mínimo que se encuentren con rendimiento académico bajo.

\section{CONCLUSIONES}

De los resultados y discusión mostrados, las conclusiones del comportamiento del consumidor en el uso de la biblioteca virtual o base de datos de acceso libre: caso de los estudiantes de la Universidad Nacional del Altiplano, son las siguientes: 1) el comportamiento del usuario de la biblioteca virtual tiene mínima influencia por la facilidad y rapidez de obtención de la información para la elaboración de sus trabajos académicos; 2) reducido número de estudiantes usan diariamente la biblioteca virtual para hacer sus trabajos académicos, pero, la mayoría usa la biblioteca virtual y físico; 3) la información virtual abundante se constituye en una ayuda para elaborar los trabajos de calidad con crítica y reflexión; 4) los autores que consultan para realizar sus trabajos reconocen mediante citas. Los resultados pueden ser extrapolados para realidades similares.

\section{AGRADECIMIENTO}

A la Universidad Nacional del Altiplano por el apoyo financiero a través del Fondo Especial de Desarrollo Universitario - FEDU.

\section{REFERENCIAS}

Abreu, Y., Domínguez, L., y Fernández, M., La biblioteca digital personalizable como complemento del sistema de bibliotecas de las universidades, Revista Cubana de ACIMED, 22(3), 262-270 (2011)

Adeniran, P., Usage of electronic resources by undergraduates at the redeemer's university, Nigeria, https://doi.org/10.5897/IJLIS2013.0392, International Journal of Library and Information Science 5(10), 319-324 (2013)

Aguilar, J.L., y Moctezuma, A., Delimitando al concepto de alfabetización: una propuesta para un mejor entendimiento, https://doi.org/10.33595/2226-1478.11.2.428, Comuni@cción: Revista de Investigación en Comunicación y Desarrollo, 11(2), 153-163 (2020)

Alaslani, K., y Alandejani, M., Identifying factors that influence students performance through social networking sites: an exploratory case study, https://doi.org/10.1016/j.heliyon.2020.e03686, Heliyon 6(4), 2-9 (2000)

Alijani, R., y Khasseh, A., Distance learners familiarity with the concept of digital library: a case study of virtual university of Shiraz, Iran, Library Philosophy and Practice, 1214 (2015) 
Bashorun, M.T., Jain, P., Sebina, P.M., y Kalusopa, T., Determinants of adoption and use of open access publishing by academic staff in Nigeria universities, https://doi.org/10.1633/JISTaP.2016.4.3.3, Journal of Information Science Theory and Practice, 4(4), 49-63 (2016)

Camacho, J.A., La biblioteca escolar en España: pasado, presente y un modelo para el futuro, Ediciones de la Torre, Madrid (2004)

Cárdenas, H., La biblioteca y la biblioteca escolar en la legislación educativa mexicana, Biblioteca Universitaria, 15(2), 147-162 (2012)

Carrera, O., Delgado, S., y otros dos autores, Factores que incentivan el uso de la biblioteca virtual en los estudiantes universitarios: un estudio de caso de la Universidad de Gómez Palacio de Durango,

https://doi.org/10.5195/biblios.2017.333, Biblios (66), 98-111 (2017)

Carter, H., Hussey, J., y Forehand, J.W., Plagiarism in nursing education and the ethical implications in practice, https://doi.org/10.1016/j.heliyon.2019.e01350, Heliyon 5, e01350, 1-8 (2019)

Eret, E., y Gokmenoglu, T., Plagiarism in higher education: a case study with prospective academicians, https://doi.org/10.1016/j.sbspro.2010.03.505, Procedia Social and Behavioral Sciences 2, 3303-3307 (2010)

Finn, K.V., y Frone, M.R., Academic performance and cheating: moderating role of school identification and self-efficacy. https://doi.org/10.3200/JOER.97.3.115-121, The Journal of Educational Research, 97(3) 115-122 (2004)

Golitsyna, I., Educational process in electronic information-educational environment, https://doi.org/10.1016/j.sbspro.2017.02.132, Procedia-Social and Behavioral Sciences, 237, 939-944 (2017)

Jiménez, C.M., Estudio sobre el estado de las webs de bibliotecas escolares en Andalucía y Extremadura y propuestas para su mejora, https://doi.org/10.1016/S0187-358X(13)72542-5, Investigación Bibliotecológica, 27(60), 27-50 (2013)

Hernández, J.S., Palomo, M.A., y Cantú, J.L., Factores endógenos y exógenos que impactan en el uso de la biblioteca en tres universidades del área metropolitana de Monterrey, Nuevo León, https://doi:10.1016/S0187-358X(13)72554-1, Investigación Bibliotecológica, 27(61), 53-81 (2013)

Marsden, H., Carroll, M., y Neill, J.T., Who cheats at university? a self-report study of dishonest academic behaviours in a sample of Australian university students, https://doi.org/10.1080/00049530412331283426, Australian Journal of Psychology, 57(1), 1-10 (2005)

Mawere, T., y Sai, K.O.S., An investigation on e-resource utilisation among university students in a developing country: a case of Great Zimbabwe University, https://doi.org/10.4102/sajim.v20i1.860, South African Journal of Information Management 20(1), 1-7 (2018)

Mazurkiewicz, O., y Sturm, T., Researching latin america, part two: a survey of how the new generation is doing its research, Investigación Bibliotecológica, 28(63), 163-192 (2014)

Meneses, F., Bibliotecas y sociedad: el paradigma social de la biblioteca pública, https://doi.org/10.1016/S0187358X(13)72558-9, Investigación Bibliotecológica, 27(61), 157-173 (2013)

Moeck, P.G., Academic dishonesty: cheating among community college studetns, community college, https://doi.org/10.1080/02776770290041846, Journal of Research and Practice, 26, 479-491 (2002)

Niño, A.L., Acercamiento a la visibilidad de la información en salud en Colombia y la biblioteca virtual de salud nacional, https://doi.org/10.1016/j.reper.2016.11.001, Repertorio de Medicina y Cirugía, 25(4), 228-231 (2016)

Osuna, C., y Luna, E., Valores éticos en la formación universitaria de las áreas de ciencias naturales e ingeniería y tecnología, en el contexto de la sociedad del conocimiento, http://dx.doi.org/10.4067/S0718-50062011000500005, Formación Universitaria, 4(5), 29-36 (2011)

Ramírez-Leyva, E.M., Encouraging reading for pleasure and the comprehensive training for readers, Investigación Bibliotecológica, 30(69), 93-116 (2016)

Romero, R., Bibliotecas virtuales y alfabetización en información, Apertura, 7(6), 92-99 (2007)

Rodríguez-García, A.A., New directions in information access, Investigación Bibliotecológica 30(69) 117-135 (2016)

Kasman, J., The virtual library, Educational Leadership, 63(4) 54-59 (2006)

Sanz-Valero, J., Juan-Quilis, V., y Ronda-Pérez E., Recursos bibliográficos disponibles en internet sobre sustancias y residuos peligrosos y su influencia en el medio ambiente en el periodo 1975 a 2005, Rev. de Salud Ambiental, 8(1), 3641 (2008)

Silva, D., Rocha, E., y otros dos autores, Comportamento de compra: principais fatores que influenciam o consumidor, Revista Científica do Itpac, 3(4), 41-47 (2010)

Xavier, T., y Godoy, A.F., Biblioteca 2.0: levantamento do seu uso em bibliotecas, Ciencias de la Información, 41(2),17$26(2010)$

Yucra, Y.J., Planificación del tiempo de estudio: caso de los estudiantes de la escuela profesional de ciencias de la comunicación-UNA Puno, Comuni@cción: Revista de Investigación en Comunicación y Desarrollo, 2(1), 62-71 (2011) 